\title{
a-Cd13-xSb10 - the devil is in the details
}

\begin{tabular}{|r|l|}
\hline Journal: & Zeitschrift für Anorganische und Allgemeine Chemie \\
\hline Manuscript ID: & zaac. 200900205.R1 \\
\hline Wiley - Manuscript type: & Article \\
\hline Date Submitted by the \\
Author: & $28-A p r-2009$ \\
\hline Complete List of Authors: & $\begin{array}{l}\text { Lidin, Sven; Stockholm university } \\
\text { Ponou, Simeon; Stockholm university, Inorganic Chemistry }\end{array}$ \\
\hline Keywords: & thermoelectric, cadmium antimonide, cadmium deficiency \\
\hline
\end{tabular}

\section{(5) ScholaroNE \\ Manuscript Central}




\title{
$\alpha-\mathrm{Cd}_{13-\mathrm{x}} \mathrm{Sb}_{10}$ - the devil is in the details
}

\author{
Simeon Ponou \\ Sven Lidin \\ Sven@inorg.su.se \\ Inorganic Chemistry \\ Arrhenius laboratory \\ Stockholm University \\ 10691 Stockholm \\ Sweden
}




\begin{abstract}
A detailed study of the structure of $\alpha-\mathrm{Cd}_{13} \mathrm{Sb}_{10}$ was performed using a high quality single crystal obtained from a Cd-rich melt. The study clearly shows that this sample is Cd-deficient, and should more properly be labelled $\alpha-\mathrm{Cd}_{13-\mathrm{x}} \mathrm{Sb}_{10}$, but whether this is true in general for this phase, or if it is an effect of the precise conditions of synthesis remains unclear. Like for the similar Zn based compound, small differences in the $\mathrm{Cd}(\mathrm{Zn})$ content may be crucial in determining the electronic transport properties of the material.
\end{abstract}




\section{Introduction}

The discovery that the compound $\mathrm{Zn}_{4} \mathrm{Sb}_{3}$ displays thermal transport properties akin to these of Perspex sparked a lot of interest in this unusual compound ${ }^{\mathrm{i}}$, since bad transport properties is a good thing in a thermoelectric material. That interstitial zinc was responsible for the extreme transport behaviour was first suggested by Snyder $e t a l .{ }^{\mathrm{ii}}$ and in a sequence of studies Häußermann et al have conclusively proven the presence of interstitials in this system, as well as a number of different ordering schemes for these $\mathrm{e}^{\mathrm{iii}, \mathrm{i}, \mathrm{v}, \mathrm{v}}$. The importance of subtle changes in composition for the physical properties of the material has been pointed out by several

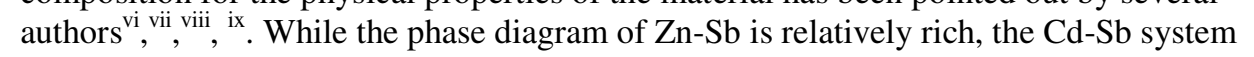
contains only one stable phase, the equimolar $\mathrm{CdSb}$, but on the $\mathrm{Cd}$ rich side of the phase diagram a number of phases may be formed with relative facility either from by undercooling.the binary melt or from ternary fluxes with $\mathrm{Bi}$, Sn or In. At least two distinct compounds can be made this way, $\mathrm{Cd}_{4} \mathrm{Sb}_{3}$ and $\mathrm{Cd}_{3} \mathrm{Sb}_{2}$, both showing temperature dependent polymorphism. We recently reported on the synthesis, structural elucidation and properties measurements of the two polymorphs of $\mathrm{Cd}_{4} \mathrm{Sb}_{3}$, the high temperature phase $\beta-\mathrm{Cd}_{4} \mathrm{Sb}_{3}$ and the room temperature phase $\alpha-\mathrm{Cd}_{4} \mathrm{Sb}_{3}{ }^{\mathrm{x}}$. The phase transition temperature depends significantly on the flux, for a quenched binary sample the $\alpha \rightarrow \beta$ transformation temperature was $375 \mathrm{~K}$, for a Sn flux grown sample the transition temperature was depressed to just above $360 \mathrm{~K}$ and for a Bi flux grown sample the transition took place below $360 \mathrm{~K}$. The hysteresis was also quite different for the three samples, for the binary melt quenched sample and the Bi flux grown sample the heating and cooling transitions differed by about $5 \mathrm{~K}$ in temperature, but for the $\mathrm{Sn}$ grown sample, the difference is more than $25 \mathrm{~K}$, even though the $\mathrm{Sn}$ content as measured by electron microprobe analysis is less than $0.1 \%$. The $\mathrm{Cd} / \mathrm{Sb}$ balance in the samples shows small differences, but lie close to the crystallographic composition of the $\alpha$ phase, $\mathrm{Cd}_{13} \mathrm{Sb}_{10}$.

The phase transition is caused by the ordering of interstitial $\mathrm{Cd}$ positions. This ordering causes some regular $\mathrm{Cd}$ sites (corresponding to Wyckoff position 36f in the trigonal $\beta$ phase cell) to be vacated. In the corresponding $\mathrm{Zn}$ based system, we have found that the $\alpha$-phase is not fully ordered, but that the ordered $\mathrm{Zn}$ interstitials are partially unoccupied, and the vacation of the corresponding regular sites is also incomplete.

The single crystal structural study of the melt quenched sample allowed for the solution of the $\alpha-\mathrm{Cd}_{4} \mathrm{Sb}_{3}$ phase super structure, but all attempts to locate residual electron density corresponding to incomplete vacation of regular sites were unsuccessful. The quality of the single crystal refinement was however limited by the quality of the single crystal specimen, and in order to further investigate possible occupational disorder in $\alpha-\mathrm{Cd}_{4} \mathrm{Sb}_{3}$ we therefore pursued a number of different synthetic procedures in order to grow better quality single crystals that might allow a high quality refinement.

\section{Experimental}

\section{Synthesis}

A single crystal of the title compound was obtained from a $\mathrm{Cd}$ rich melt. A mixture of approximately $1 \mathrm{~g}$ of the elements $(\mathrm{Cd}, 99.9 \%$ and $\mathrm{Sb}, 99.5 \%$; all purchased from SigmaAldrich) with the weight ratio $\mathrm{Cd}: \mathrm{Sb}=0.65: 0.40$ was loaded in a steal ampoule which was weld-sealed at both ends under argon atmosphere. The sample was first placed vertically in a muffle oven and heated at $923 \mathrm{~K}$. Then, the ampoule was turned to a horizontal position and the oven temperature was cooled down to $673 \mathrm{~K}$ where the sample was subsequently annealed 
for about 48 hours. Finally, the sample was removed from the furnace and allowed to cool down to room temperature.

\section{Single crystal $X$-ray diffraction}

A suitable crystal was picked from the crushed sample, mounted on a glass fibre and diffraction data was measured on an Oxford Diffraction XCalibur 3 CCD diffractometer with MoKa radiation $(\lambda=0.71073 \AA$. $50 \mathrm{kV}, 40 \mathrm{~mA})$. Two data sets were acquired at room temperature $295 \mathrm{~K}$ and at $100 \mathrm{~K}$ using an Oxford cryostream system. The data reduction was performed using the Oxford diffraction Crysalis system, and the refinement was carried out using the JANA2000 program ${ }^{\mathrm{xi}}$. All details of the measurements are given in tables 1 and 2.The monoclinic super structure cell is obvious in the diffraction pattern, but the small crystal chosen exhibits less obvious twinning than expected, and picking out a dominant domain was straight-forward. In figure 1 the twinning pattern is indicated. As indicated, the diffraction pattern consists of two distinct sets. The basic reflections of the $\beta$-phase are common to all individuals, while the satellites are unique to each of the three domain orientations in the twinned structure. The data is therefore readily deconvoluted, although absolute intensity information is available only after the structure is solved and refined. In practise, as explained in previous work, to solve the structure a model featuring the $\beta$-phase in the setting of the monoclinic unit cell and embellished with a number of interstitial positions identified as noticeable in the electron density map of the solution from the $\beta$-phase was used as a starting set. This model was then refined by allowing parial occupancy for any atoms that had neighbours at unphysically short distances. After a number of least squares refinement cycles, positions with an occupancy below a threshold walue were discarded, the difference electron density map was evaluated to identify new possible interstitial positions, and the distance map was re-evaluated to identify which positions that should be refined in terms of occupancy. The threshold value was adjusted during the refinement to yield increasingly hard conditions for considering a position as empty. This solution strategy was used in the maximal monoclinic subgroups to $R-3 c, P 2_{1} / n$, and subsequently in the three subgroups $P n, P 2_{1}$, and $P$ 1. A satisfactory convergence was achievable only in $P n$. The structure in space group $P n$ from previous work was used as a starting model with 60 independent $\mathrm{Sb}$ positions and 78 independent $\mathrm{Cd}$ positions. The $\mathrm{Sb}$ atoms were grouped in two sets ( $\mathrm{A}$ and $\mathrm{B}$ ) according to their positions in the $\beta$ phase structure. The $\mathrm{Cd}$ positions were also grouped in two sets, a first, larger, set of $64 \mathrm{Cd}$ positions (A) corresponding to the $36 \mathrm{f}$ position in the $\beta$ phase, and one grouping consisting of the 14 ordered interstitial Cd positions (B). Within these groups each set was modelled using a single, isotropic, thermal displacement parameter. After convergence of this refinement, the difference electron density map was examined for prominent residuals, and the positions of these were compared to, and found to correspond to, those of the vacated regular $\mathrm{Cd}$ positions. A new set $(\mathrm{C})$ of these 8 positions were included as partially occupied in a modified model, and the occupancy of the $14 \mathrm{Cd}$ positions in group B corresponding to the ordered interstitials was reduced by the same amount. In the ensuing cycles of refinement the occupancies of these two groups were coupled complementarily. Since the 8 new positions correspond to the $36 \mathrm{f}$ position in the $\beta$ phase, the isotropic thermal displacement parameter of group $\mathrm{CdC}$ was set equal to that of set $\mathrm{CdA}$. All pertinent parameters for the $\mathrm{x}$ ray study are given in table 1 and 2 . Final positional and occupational parameters are given in tables 3 and 4 .

\section{Results and discussion}


The results of the refinements, both for the data collected at ambient temperature and for the data from $100 \mathrm{~K}$ largely agree with the results previously published, but with two important exceptions. First, the quality of the crystal, and hence the data is far superior in this measurement compared to that of the crystals used earlier, and this is also clear from the final agreement between model and data. The number of observed reflections is lower, as a result of the smaller sample size, but the parameter-to-data ratio is still close to 10 even for the observed $\left(\mathrm{F}^{2}>3 \sigma\left(\mathrm{F}^{2}\right)\right)$ set. The volumes of the minor twin constituents were about $1 / 8$ each, adding up to $1 / 4$ of the total crystal volume. The twinning matrices were as reported earlier

$\begin{array}{rrr}0.5000 & -1.0000 & 1.5000 \\ 0.2500 & -0.5000 & -0.7500 \\ 0.3333 & 0.6667 & 0.0000 \\ & & \\ 0.4971 & 1.0000 & 1.5000 \\ -0.2500 & -0.5000 & 0.7500 \\ 0.3333 & -0.6667 & 0.0000\end{array}$

As shown earlier, the ordered interstitials form chains along the monoclinic a-axis. In figure

2a, the relative positions of these chains are shown in a projection parallel to $\mathbf{a}$. Sb atoms are shown in blue, set $A$ of the $\bar{C}$ atoms is shown in yellow, set $B$ in red and set $C$ in green. It is evident that the chains are well separated and that the deformation of the surrounding

| structure is negligible. A view down the monoclinic c-axis (fig $2 b$ ) is ideal for displaying the structure of the chain of interstitials. In this view, the atomic positions of set $\overline{\mathrm{S}} \overline{\mathrm{A}}$ and $\overline{\mathrm{S}} \overline{\mathrm{B}}$ and set $\mathrm{CdA}$ have been removed from the top of the image to display the chain of interstitials of group $\mathrm{CdB}$ and the alternative positions of group $\mathrm{CdC}$ more clearly. Finally, in the image

2 $2 \mathrm{c}$, where the structure is projected down the b-axis, the lamellar nature of the structure is shown. An alternation between two filled and one empty lamella is obvious, and this is clearly the reason for the 3 -fold superstructure along $\mathrm{c}$.

The reduction in Cd content, $\mathrm{x}$, generated by the partial exchange of the 14 atomic positions in group $\mathrm{CdB}$ for the 8 positions in group $\mathrm{CdC}$ is given by $\mathrm{Cd}(\mathrm{A})_{64} \mathrm{Cd}(\mathrm{B})_{14(1-\mathrm{x})} \mathrm{Cd}(\mathrm{C})_{8 \mathrm{x}} \mathrm{Sb}(\mathrm{A}, \mathrm{B})_{60}$ which simplifies to $\mathrm{Cd}_{13-\mathrm{x}} \mathrm{Sb}_{10}$. In the final refinement, the deficiency on the occupancy of set $\mathrm{CdC}$ converges to about $20 \%$. This means that the $\mathrm{Cd}$ content is reduced to $\mathrm{Cd}_{12.8} \mathrm{Sb}_{10}$, or from $56.5 \%$ to $56.1 \%$, equivalent to about two esd. in the electron microprobe analysis. The failure to locate significant electron density on positions corresponding to set $\mathrm{CdC}$ in our previous work may be taken as an indication that this product was in fact stoichiometric $\mathrm{Cd}_{13} \mathrm{Sb}_{10}$, but as mentioned in the introduction, it may equally well simply reflect the quality of the single crystal. It should be noted that weak super structure ordering combined with twinning places rather high demands on crystal quality for detailed analysis. We therefore cannot say with any certainty that the two samples really differ in composition, but there are certainly good reasons to assume that a certain composition range is permissible for $\mathrm{Cd}_{4} \mathrm{Sb}_{3}$. The crystal used in this study has a unit cell volume that is $0.2 \%$ smaller (at ambient conditions and $0.1 \%$ at $100 \mathrm{~K}$ ) than that used in the previous study. The significance of this difference is debatable, and it is in fact larger than would be expected from the $\mathrm{Cd}$ deficiency alone. The fact that the phase may be grown from a rather extended region, compositionally as well as in temperature, and that the phase originally formed is the disordered $\beta$-phase certainly leaves room for compositional variations. The local structure of $\alpha-\mathrm{Cd}_{4} \mathrm{Sb}_{3}$ comprising lamellae containing ordered interstitials interleaved with lamellae devoid of interstitial positions certainly offers a facile way to structurally accommodate a slight variation in under-occupancy of $\mathrm{Cd}$. This is indeed the case for the analogous $\mathrm{Zn}_{4} \mathrm{Sb}_{3}$ 
compound which further strengthens the suspicion. A last important indication is the report by Zelinska ${ }^{\text {xii }}$ of $\mathrm{a} \mathrm{Cd}_{4} \mathrm{Sb}_{3}$ quenched from the binary melt that doesn't exhibit any super structure ordering at room temperature. This would be most easily understood in terms of small compositional variations determining the transition temperature between the $\alpha$ and $\beta$ forms of this phase.

\section{Conclusion}

Formatted: Font: Bold

Ths $\alpha$-phase structure of $\mathrm{Cd}_{13} \mathrm{Sb}_{10}$ from a new synthetic protocol was unambiguously shown to be $\mathrm{Cd}$ deficient, i.e., the compound is $\bar{C} \mathrm{~d}_{13}-\mathrm{S} \mathrm{S}_{10}$. This does not mean that previous studies by us and others indicating a stoichiometric $\alpha$-phase, or even a room temperature $\beta$-phase are in error. To the contrary, we think it indicates a compositional variability within certain bounds that allows for a Cd-poor sample to remain in the $\beta$-phase throughout the entire temperature range of the investigation, or to display various degrees of $\alpha / \beta$-polyphasic behaviour. As for the compound $\mathrm{Zn}_{13} \underline{S b}_{10}$, the $\alpha$-phase super structure displays a clear layer structure that allows for the facile intergrowth between the two phases. The fact that the $\overline{\mathrm{Cd}}$ compound only forms as a meta stable compound from the melt make detailed investigations of compositional and temporal parameters difficult. Slow cooling of a melt always results in elemental $\mathrm{Cd}$ and the $1: 1$ compound.

\section{Acknowledgement}

Formatted: Font: Bold

Financial support from the Swedish research council and the Wennergren Foundation (post doc financing for S.P.) in gratefully acknowledged. This work is part of the effort within the CMA European project. 
1

2

3

4

5

6

7

8

9

10

11

12

13

14

15

16

17

18

19

20

21

Figure captions

1

Scheme of the twinned diffraction pattern. The reflections marked by red circles are main reflections from the high temperature b-phase, while those marked by yellow cicles generate the superstructure. Every red reflection is part of three twin lattices as indicated for the set along the $\mathrm{c}^{*}$ axis by the three intersection planes.

The structure of the compound $\mathrm{Cd}_{13-x} \mathrm{Sb}_{10}$ in axial projection. Sb positions are shown in blue, regular $\mathrm{Zn}$ positions in yellow, ordered interstitial $\mathrm{Zn}$ positions are shown in red and finally partially occupied regular $\mathrm{Zn}$ in green. a) view down a-axis, b) view down c-axis, c) view down b-axis 
Table 1

Crystal data

\begin{tabular}{l|l}
$\underline{\mathrm{Cd}}_{153.662} \underline{\mathrm{Sb}_{120}}$ & $V=\underline{7643.2(5)} \AA^{3}$ \\
\hline$M_{r}=\underline{31883.1}$ & $Z=\underline{1}$ \\
\hline$\underline{\text { Monoclinic }, \underline{P n}}$ & $\underline{\text { Mo } K \alpha}$ \\
\hline$a=\underline{11.4478(4)} \AA$ & $\mu=\underline{20.81 \mathrm{~mm}^{-1}}$ \\
\hline$b=\underline{26.0793(7)} \AA$ & $T=\underline{293} \mathrm{~K}$ \\
\hline$c=\underline{26.0691(10)} \AA$ & $\underline{0.10} \times \underline{0.05} \times \underline{0.03} \mathrm{~mm}$ \\
\hline$\beta=\underline{100.872(4)}$ & \\
\hline
\end{tabular}

Data collection

XCalibur 3 diffractometer

Absorption correction: Gaussian

CrysAlis RED, Oxford Diffraction Ltd.,

Version 1.171.32.9 (release 17-07-2007

CrysAlis171 .NET) (compiled Aug 17

2007,17:36:27) Numerical absorption

correction based on Gaussian integration over a multifaceted crystal model

$T_{\min }=\underline{0.288}, T_{\max }=\underline{0.656}$

$R_{\text {int }}=\underline{0.175}$ $\underline{42170}$ independent reflections

$\underline{2407}$ reflections with $\underline{I>3 \sigma(I)}$

Deleted: $g$

$\underline{72678}$ measured reflections

Refinement

\begin{tabular}{|c|c|}
\hline $\begin{array}{l}R\left[F^{2}>2 \sigma\left(F^{2}\right)\right]= \\
\underline{0.038}\end{array}$ & $\Delta \rho_{\max }=\underline{5.10} \mathrm{e} \AA^{-3}$ \\
\hline$w R\left(F^{2}\right)=\underline{0.070}$ & $\Delta \rho_{\min }=-3.82 \mathrm{e} \AA^{-3}$ \\
\hline$S=\underline{0.50}$ & \\
\hline$\underline{42170}$ reflections & $\underline{445}$ parameters \\
\hline
\end{tabular}


Table 2

\section{Crystal data}

\begin{tabular}{|c|c|}
\hline$\underline{\mathrm{Cd}}_{154.036} \underline{\mathrm{Sb}}_{120}$ & $V=\underline{7636.3(5)} \AA^{3}$ \\
\hline$M_{r}=\underline{31925.2}$ & $Z=\underline{1}$ \\
\hline$\underline{\text { Monoclinic, }} \underline{P n}$ & $\underline{\operatorname{Mo} K \alpha}$ \\
\hline$a=\underline{11.4469(4)} \AA$ & $\mu=\underline{20.85} \mathrm{~mm}^{-1}$ \\
\hline$b=\underline{26.0624(9)} \AA$ & $T=\underline{100} \mathrm{~K}$ \\
\hline$c=\underline{26.0579(10)} \AA$ & $\underline{0.10} \times \underline{0.05} \times \underline{0.03} \mathrm{~mm}$ \\
\hline$\beta=\underline{100.797(3)^{\circ}}$ & \\
\hline
\end{tabular}

\section{Data collection}

\section{XCalibur 3}

diffractometer diffractometer $\underline{42010}$ independent reflections

$\underline{3243}$ reflections with $\underline{I>3 \sigma(I)}$

\section{Absorption correction: Gaussian \\ CrysAlis RED, Oxford Diffraction Ltd., \\ Version 1.171.32.9 (release 17-07-2007 \\ CrysAlis171 .NET) (compiled Aug 17 \\ 2007,17:36:27) Numerical absorption \\ correction based on Gaussian integration over a multifaceted crystal model}
$T_{\min }=\underline{0.288}, T_{\max }=\underline{0.656}$
$R_{\text {int }}=\underline{0.144}$

\section{Refinement}

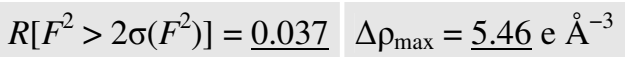

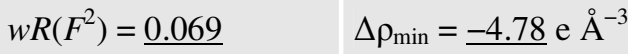

$$
\begin{aligned}
& S=\underline{0.56} \\
& \underline{42010} \text { reflections } \quad \underline{45} \text { parameters }
\end{aligned}
$$


Table 3

Fractional atomic coordinates and isotropic or equivalent isotropic displacement parameters $\underline{(\AA 2)}$ at $\mathrm{T}=293 \mathrm{~K}$

\begin{tabular}{|c|c|c|c|c|c|}
\hline & $x$ & $y$ & $z$ & $U_{\text {iso }} * / U_{\text {eq }}$ & Occ. $(<1)$ \\
\hline Sba1 & 1.600263 & 0.8777 & 0.692587 & $0.02009(10)^{*}$ & \\
\hline Sba2 & $2.0982(12)$ & $0.6292(3)$ & $0.6974(4)$ & $0.02009(10)^{*}$ & \\
\hline Sba3 & $1.5962(11)$ & $0.8729(3)$ & $0.3664(4)$ & $0.02009(10)^{*}$ & \\
\hline Sba4 & $1.6062(12)$ & $0.6167(3)$ & $0.8571(5)$ & $0.02009(10)^{*}$ & \\
\hline Sba5 & $2.0964(11)$ & 0.8745 & $0.5277(4)$ & $0.02009(10)^{*}$ & \\
\hline Sba6 & 2.1017 (13) & $1.1259(3)$ & $0.3592(5)$ & $0.02009(10)^{*}$ & \\
\hline Sba7 & 1.1013 (13) & 0.6213 & $1.0323(5)$ & $0.02009(10)^{*}$ & \\
\hline Sba8 & $1.5956(13)$ & $1.1247(3)$ & $0.5280(5)$ & $0.02009(10)^{*}$ & \\
\hline Sba9 & $1.1033(11)$ & $1.3728(3)$ & $0.5282(4)$ & $0.02009(10)^{*}$ & \\
\hline Sba10 & $1.5982(10)$ & $1.3637(3)$ & $0.3606(4)$ & $0.02009(10)^{*}$ & \\
\hline Sba11 & 1.0973 (13) & 1.1193 & $0.6947(5)$ & $0.02009(10)^{*}$ & \\
\hline Sba12 & $1.3807(12)$ & $0.6231(3)$ & $0.7893(4)$ & $0.02009(10)^{*}$ & \\
\hline Sba13 & $1.3813(11)$ & $1.1247(3)$ & $0.4551(4)$ & $0.02009(10)^{*}$ & \\
\hline Sba14 & $1.3831(12)$ & 0.8791 & $0.6245(4)$ & $0.02009(10)^{*}$ & \\
\hline Sba15 & $1.3800(12)$ & $1.3705(3)$ & $0.2873(4)$ & $0.02009(10)^{*}$ & \\
\hline Sba16 & $1.8832(11)$ & $0.6267(3)$ & $0.6249(4)$ & $0.02009(10)^{*}$ & \\
\hline Sba17 & $1.8843(9)$ & 0.8745 & 0.4535 & $0.02009(10)^{*}$ & \\
\hline Sba18 & $0.8834(12)$ & $1.1220(3)$ & $0.6172(4)$ & $0.02009(10)^{*}$ & \\
\hline Sba19 & 0.8837 (11) & 0.6151 & $0.9580(4)$ & $0.02009(10)^{*}$ & \\
\hline Sba20 & $1.3816(12)$ & 0.8706 & $0.2885(4)$ & $0.02009(10)^{*}$ & \\
\hline Sba21 & $0.8806(12)$ & $1.3733(3)$ & $0.4593(4)$ & $0.02009(10)^{*}$ & \\
\hline Sba22 & $1.3815(12)$ & 0.3759 (3) & $0.6222(4)$ & $0.02009(10)^{*}$ & \\
\hline $\mathrm{Sba} 23$ & $1.8804(11)$ & $1.1250(3)$ & $0.2872(4)$ & $0.02009(10)^{*}$ & \\
\hline Sba24 & $1.6003(11)$ & 0.3807 (3) & $0.6941(4)$ & $0.02009(10)^{*}$ & \\
\hline Sbb25 & $1.8167(11)$ & 0.5377 & $0.7736(4)$ & $0.01987(9)^{*}$ & \\
\hline Sbb26 & $1.6743(11)$ & $0.7129(3)$ & $0.7077(4)$ & $0.01987(9)^{*}$ & \\
\hline
\end{tabular}




\begin{tabular}{|c|c|c|c|c|}
\hline Sbb27 & $1.4969(11)$ & $0.9485(3)$ & 0.4895 (4) & $0.01987(9)^{*}$ \\
\hline Sbb28 & $1.3117(11)$ & $1.2791(3)$ & $0.4480(4)$ & $0.01987(9)^{*}$ \\
\hline Sbb29 & $1.3223(11)$ & $0.7184(3)$ & 0.6133 (4) & $0.01987(9)^{*}$ \\
\hline Sbb30 & $1.8090(11)$ & $0.9618(3)$ & $0.6062(4)$ & $0.01987(9)^{*}$ \\
\hline Sbb31 & $1.4919(11)$ & $0.5469(3)$ & 0.9904 (4) & $0.01987(9)^{*}$ \\
\hline Sbb32 & $2.1671(12)$ & $0.5360(3)$ & $0.8715(4)$ & $0.01987(9)^{*}$ \\
\hline Sbb33 & 1.9907 (11) & $0.8012(3)$ & $0.6580(4)$ & $0.01987(9)^{*}$ \\
\hline Sbb34 & $1.3112(11)$ & $0.7900(3)$ & $0.7744(4)$ & $0.01987(9)^{*}$ \\
\hline Sbb35 & $1.3192(11)$ & $1.2096(3)$ & $0.6036(4)$ & $0.01987(9)^{*}$ \\
\hline Sbb36 & $1.9919(11)$ & $0.6965(3)$ & 0.8241 (4) & $0.01987(9)^{*}$ \\
\hline Sbb37 & $1.9891(12)$ & $1.2953(3)$ & $0.3255(4)$ & $0.01987(9)^{*}$ \\
\hline Sbb38 & $1.4955(11)$ & $0.5579(3)$ & $0.6544(4)$ & $0.01987(9)^{*}$ \\
\hline Sbb39 & $0.9938(11)$ & $1.1950(3)$ & $0.4913(4)$ & $0.01987(9)^{*}$ \\
\hline Sbb40 & $2.1658(11)$ & $0.5420(3)$ & $0.5434(4)$ & $0.01987(9)^{*}$ \\
\hline Sbb41 & $1.6738(11)$ & $0.7818(3)$ & 0.5409 (4) & $0.01987(9)^{*}$ \\
\hline Sbb42 & $1.2950(10)$ & $0.7154(3)$ & $0.9510(4)$ & $0.01987(9)^{*}$ \\
\hline Sbb43 & $1.4909(11)$ & $1.0487(3)$ & 0.6549 (4) & $0.01987(9)^{*}$ \\
\hline Sbb44 & $1.4951(11)$ & $0.4545(3)$ & 0.8217 (4) & $0.01987(9)^{*}$ \\
\hline Sbb45 & $1.8183(11)$ & $1.0380(3)$ & $0.4372(4)$ & $0.01987(9)^{*}$ \\
\hline Sbb46 & $1.6787(11)$ & $0.7815(3)$ & $0.8702(4)$ & $0.01987(9)^{*}$ \\
\hline Sbb47 & $1.4909(11)$ & $1.0550(3)$ & $0.3223(4)$ & $0.01987(9)^{*}$ \\
\hline Sbb48 & $2.1601(11)$ & $0.9688(3)$ & $0.7012(4)$ & $0.01987(9)^{*}$ \\
\hline Sbb49 & $1.6683(11)$ & $1.2887(3)$ & 0.5417 (4) & $0.01987(9)^{*}$ \\
\hline Sbb50 & $1.7974(11)$ & $0.9584(3)$ & $0.2783(4)$ & $0.01987(9)^{*}$ \\
\hline Sbb51 & $1.8153(11)$ & $0.4652(3)$ & 0.9497 (4) & $0.01987(9)^{*}$ \\
\hline Sbb52 & $0.9932(12)$ & $1.3038(3)$ & 0.6564 (4) & $0.01987(9)^{*}$ \\
\hline Sbb53 & 1.4917 (12) & $1.4504(3)$ & 0.4913 (4) & $0.01987(9)^{*}$ \\
\hline Sbb54 & $2.1655(11)$ & $0.9591(3)$ & $0.3790(4)$ & $0.01987(9)^{*}$ \\
\hline Sbb55 & $1.8089(11)$ & $0.4615(3)$ & 0.6094 (4) & $0.01987(9)^{*}$ \\
\hline Sbb56 & $2.1619(11)$ & $0.4642(3)$ & 0.7057 (4) & $0.01987(9)^{*}$ \\
\hline
\end{tabular}




\begin{tabular}{|c|c|c|c|c|}
\hline Sbb57 & $2.1638(11)$ & $1.0324(3)$ & $0.5334(4)$ & $0.01987(9)^{*}$ \\
\hline Sbb58 & $1.6753(11)$ & $1.2133(3)$ & $0.7063(4)$ & $0.01987(9)^{*}$ \\
\hline Sbb59 & $1.6725(11)$ & $1.2115(3)$ & $0.3716(4)$ & $0.01987(9)^{*}$ \\
\hline Sbb60 & $1.8147(12)$ & $1.0348(3)$ & $0.7778(4)$ & $0.01987(9)^{*}$ \\
\hline Cda1 & $1.5679(13)$ & $0.7974(4)$ & $0.7613(5)$ & $0.02839(9)^{*}$ \\
\hline $\mathrm{Cda} 2$ & $2.1032(13)$ & $0.7285(3)$ & $0.7347(5)$ & $0.02839(9)^{*}$ \\
\hline $\mathrm{Cda} 3$ & $2.0595(12)$ & $0.5515(3)$ & $0.7643(5)$ & $0.02839(9)^{*}$ \\
\hline Cda4 & $1.6164(13)$ & $0.9734(4)$ & $0.4042(5)$ & $0.02839(9)^{*}$ \\
\hline $\mathrm{Cda} 5$ & $1.5663(13)$ & $1.2033(4)$ & $0.5948(5)$ & $0.02839(9)^{*}$ \\
\hline Cda6 & $1.7664(12)$ & $0.6496(3)$ & $0.7971(5)$ & $0.02839(9)^{*}$ \\
\hline Cda7 & $2.0617(13)$ & $1.0474(3)$ & $0.4263(5)$ & $0.02839(9)^{*}$ \\
\hline Cda8 & $1.6172(13)$ & $0.5225(4)$ & $0.9039(5)$ & $0.02839(9)^{*}$ \\
\hline Cda9 & $1.1013(12)$ & $1.2708(3)$ & $0.5687(5)$ & $0.02839(9)^{*}$ \\
\hline Cda10 & $2.0589(12)$ & $0.9520(3)$ & $0.5930(5)$ & $0.02839(9)^{*}$ \\
\hline Cda11 & $1.6086(13)$ & $1.0247(4)$ & $0.5672(5)$ & $0.02839(9)^{*}$ \\
\hline Cda12 & $1.7661(13)$ & $0.8540(4)$ & $0.6303(5)$ & $0.02839(9)^{*}$ \\
\hline Cda13 & $1.7696(13)$ & $1.1468(4)$ & $0.4662(5)$ & $0.02839(9)^{*}$ \\
\hline Cda14 & $1.6101(13)$ & $0.4800(4)$ & $0.7313(5)$ & $0.02839(9)^{*}$ \\
\hline Cda15 & $1.4246(13)$ & $0.6991(3)$ & $0.7217(5)$ & $0.02839(9)^{*}$ \\
\hline Cda16 & $1.8687(13)$ & 0.7759 (4) & $0.7450(5)$ & $0.02839(9)^{*}$ \\
\hline Cda17 & $1.5726(13)$ & $0.7948(3)$ & $0.4358(5)$ & $0.02839(9)^{*}$ \\
\hline Cda18 & $1.2557(12)$ & $1.0988(3)$ & $0.6214(4)$ & $0.02839(9)^{*}$ \\
\hline Cda19 & 1.3785 (12) & $0.4754(4)$ & $0.9130(5)$ & $0.02839(9)^{*}$ \\
\hline Cda20 & $1.5655(12)$ & $1.2935(3)$ & $0.4333(5)$ & $0.02839(9)^{*}$ \\
\hline $\mathrm{Cda} 21$ & $1.3818(12)$ & $1.0232(3)$ & $0.4170(5)$ & $0.02839(9)^{*}$ \\
\hline $\mathrm{Cda} 22$ & $1.2474(11)$ & $0.5825(3)$ & $0.9694(4)$ & $0.02839(9)^{*}$ \\
\hline Cda23 & $1.0689(12)$ & $0.5446(3)$ & $1.1028(5)$ & $0.02839(9)^{*}$ \\
\hline $\mathrm{Cda} 24$ & $2.2705(12)$ & $0.6070(4)$ & $0.6318(5)$ & $0.02839(9)^{*}$ \\
\hline $\mathrm{Cda} 25$ & $1.2728(13)$ & $1.3942(3)$ & $0.4650(5)$ & $0.02839(9)^{*}$ \\
\hline $\mathrm{Cda} 26$ & $1.7486(12)$ & $1.3426(3)$ & $0.2909(5)$ & $0.02839(9)^{*}$ \\
\hline
\end{tabular}




\begin{tabular}{|c|c|c|c|c|}
\hline $\mathrm{Cda} 27$ & $2.2764(13)$ & $0.8944(3)$ & $0.4704(4)$ & $0.02839(9)^{*}$ \\
\hline Cda28 & $0.8649(12)$ & $1.2231(3)$ & $0.5745(4)$ & $0.02839(9)^{*}$ \\
\hline Cda29 & $2.1166(13)$ & $1.2242(4)$ & $0.4057(5)$ & $0.02839(9)^{*}$ \\
\hline Cda30 & $1.7174(13)$ & $0.6045(4)$ & $0.6877(5)$ & $0.02839(9)^{*}$ \\
\hline Cda31 & 1.9198 (12) & $0.5373(3)$ & $0.8816(4)$ & $0.02839(9)^{*}$ \\
\hline Cda32 & $2.1166(12)$ & $0.7753(3)$ & $0.5705(4)$ & $0.02839(9)^{*}$ \\
\hline Cda33 & $1.3638(12)$ & $0.9737(3)$ & $0.5729(4)$ & $0.02839(9)^{*}$ \\
\hline Cda34 & $1.9123(12)$ & $0.9546(4)$ & $0.3883(5)$ & $0.02839(9)^{*}$ \\
\hline Cda35 & $1.2073(12)$ & $1.1410(3)$ & $0.5137(4)$ & $0.02839(9)^{*}$ \\
\hline Cda36 & $2.2152(13)$ & $0.6458(4)$ & $0.8537(5)$ & $0.02839(9)^{*}$ \\
\hline Cda37 & $1.7191(13)$ & $0.8947(4)$ & $0.5208(5)$ & $0.02839(9)^{*}$ \\
\hline Cda38 & $1.3701(12)$ & $0.5255(4)$ & $0.7400(5)$ & $0.02839(9)^{*}$ \\
\hline Cda39 & $1.9150(12)$ & $0.4637(3)$ & $0.7151(5)$ & $0.02839(9)^{*}$ \\
\hline Cda40 & 1.4207 (13) & $1.2976(3)$ & $0.5535(5)$ & $0.02839(9)^{*}$ \\
\hline Cda41 & $1.6142(13)$ & $0.9748(4)$ & $0.7366(5)$ & $0.02839(9)^{*}$ \\
\hline $\mathrm{Cda} 42$ & $1.8776(13)$ & $1.2699(4)$ & $0.4192(5)$ & $0.02839(9)^{*}$ \\
\hline Cda43 & $2.2140(12)$ & $0.8580(3)$ & $0.6873(5)$ & $0.02839(9)^{*}$ \\
\hline Cda44 & $1.9150(12)$ & $1.0400(3)$ & $0.5477(5)$ & $0.02839(9)^{*}$ \\
\hline $\mathrm{Cda} 45$ & $1.0648(13)$ & $1.4509(3)$ & $0.5971(5)$ & $0.02839(9)^{*}$ \\
\hline Cda46 & $1.2366(11)$ & $0.6634(3)$ & $0.5224(4)$ & $0.02839(9)^{*}$ \\
\hline Cda47 & $1.1125(12)$ & $0.7252(3)$ & $1.0707(4)$ & $0.02839(9)^{*}$ \\
\hline Cda48 & $1.2266(12)$ & $1.3472(3)$ & $0.3536(5)$ & $0.02839(9)^{*}$ \\
\hline Cda49 & $2.2784(12)$ & $1.1084(3)$ & $0.2944(5)$ & $0.02839(9)^{*}$ \\
\hline Cda50 & $1.0645(12)$ & $1.0453(3)$ & $0.7667(5)$ & $0.02839(9)^{*}$ \\
\hline Cda51 & $1.3816(12)$ & $0.5230(3)$ & $1.0874(5)$ & $0.02839(9)^{*}$ \\
\hline Cda52 & $1.7112(12)$ & $0.8902(3)$ & $0.1897(5)$ & $0.02839(9)^{*}$ \\
\hline Cda53 & $1.4242(13)$ & $1.2023(4)$ & $0.3862(5)$ & $0.02839(9)^{*}$ \\
\hline Cda54 & $1.7482(12)$ & $0.8369(3)$ & $0.2999(5)$ & $0.02839(9)^{*}$ \\
\hline Cda55 & $1.7735(12)$ & $0.3541(3)$ & $0.6282(5)$ & $0.02839(9)^{*}$ \\
\hline Cda56 & $1.7296(12)$ & $0.4035(3)$ & $0.8602(5)$ & $0.02839(9)^{*}$ \\
\hline
\end{tabular}




\begin{tabular}{|c|c|c|c|c|c|}
\hline Cda57 & $1.7094(12)$ & $1.1054(4)$ & $0.3530(5)$ & $0.02839(9)^{*}$ & \\
\hline Cda58 & $1.8701(13)$ & $1.2277(4)$ & $0.2421(5)$ & $0.02839(9)^{*}$ & \\
\hline Cda59 & $1.9094(12)$ & $0.9587(3)$ & $0.7131(5)$ & $0.02839(9)^{*}$ & \\
\hline Cda60 & $1.3747(12)$ & $0.4761(3)$ & $0.5834(5)$ & $0.02839(9)^{*}$ & \\
\hline Cda61 & $1.7216(12)$ & $1.4008(3)$ & $0.5192(5)$ & $0.02839(9)^{*}$ & \\
\hline Cda62 & $1.9190(12)$ & $0.5449(3)$ & $0.5568(5)$ & $0.02839(9)^{*}$ & \\
\hline Cda63 & $1.7265(12)$ & $1.0975(3)$ & $0.6842(5)$ & $0.02839(9)^{*}$ & \\
\hline Cda64 & $1.4328(12)$ & $0.8010(3)$ & $0.5562(5)$ & $0.02839(9)^{*}$ & \\
\hline Cdb65 & $0.9529(12)$ & $1.2065(4)$ & $0.7069(5)$ & $0.0212(5)^{*}$ & $0.805 \quad(3)$ \\
\hline Cdb66 & $1.5262(12)$ & $0.3475(3)$ & $0.7911(4)$ & $0.0212(5)^{*}$ & $0.805 \quad(3)$ \\
\hline Cdb67 & $1.5442(13)$ & $0.9573(4)$ & $0.2759(5)$ & $0.0212(5)^{*}$ & $0.805 \quad(3)$ \\
\hline Cdb68 & $1.2434(11)$ & $0.2043(3)$ & $0.7116(4)$ & $0.0212(5)^{*}$ & $0.805 \quad(3)$ \\
\hline Cdb69 & $1.4934(11)$ & $0.1482(3)$ & $0.7061(4)$ & $0.0212(5)^{*}$ & $0.805 \quad(3)$ \\
\hline $\mathrm{Cdb} 70$ & $1.4621(11)$ & $0.2756(3)$ & $0.6976(4)$ & $0.0212(5)^{*}$ & $0.805 \quad$ (3) \\
\hline $\mathrm{Cdb} 71$ & $1.0437(13)$ & $0.7074(4)$ & $0.9445(5)$ & $0.0212(5)^{*}$ & $0.805 \quad$ (3) \\
\hline $\mathrm{Cdb} 72$ & $1.4513(13)$ & $1.4564(4)$ & $0.3740(5)$ & $0.0212(5)^{*}$ & $0.805 \quad(3)$ \\
\hline $\mathrm{Cdb} 73$ & $1.5278(11)$ & $0.6524(3)$ & $0.9598(4)$ & $0.0212(5)^{*}$ & $0.805 \quad(3)$ \\
\hline $\mathrm{Cdb} 74$ & $1.4833(12)$ & 0.8499 (4) & $0.8707(4)$ & $0.0212(5)^{*}$ & $0.805 \quad(3)$ \\
\hline Cdb75 & $1.4641(11)$ & $0.7242(3)$ & $0.8671(4)$ & $0.0212(5)^{*}$ & $0.805 \quad(3)$ \\
\hline Cdb76 & $1.2544(11)$ & $0.8006(3)$ & $0.8860(4)$ & $0.0212(5)^{*}$ & $0.805 \quad$ (3) \\
\hline $\mathrm{Cdb} 77$ & $1.2713(12)$ & $0.9712(4)$ & $0.2909(5)$ & $0.0212(5)^{*}$ & $0.805 \quad(3)$ \\
\hline $\mathrm{Cdb} 78$ & $1.2736(12)$ & $0.5282(3)$ & $0.4594(4)$ & $0.0212(5)^{*}$ & $0.805 \quad(3)$ \\
\hline Cdc79 & $-1.491(3)$ & $0.6737(12)$ & $-0.0717(12)$ & $0.020(3)^{*}$ & $0.195 \quad$ (3) \\
\hline Cdc80 & $-1.510(3)$ & $0.2921(10)$ & $-0.2642(11)$ & $0.020(3)^{*}$ & $0.195 \quad(3)$ \\
\hline Cdc81 & $-1.228(3)$ & $1.0627(10)$ & $0.1363(11)$ & $0.020(3)^{*}$ & $0.195 \quad$ (3) \\
\hline $\mathrm{Cdc} 82$ & $-1.152(3)$ & $0.2624(11)$ & $-0.2267(12)$ & $0.020(3)^{*}$ & $0.195 \quad(3)$ \\
\hline $\mathrm{Cdc} 83$ & $1.668(3)$ & $1.0232(11)$ & 0.2107 (12) & $0.020(3)^{*}$ & $0.195 \quad$ (3) \\
\hline Cdc84 & $0.445(3)$ & $0.8213(12)$ & $0.8604(12)$ & $0.020(3)^{*}$ & $0.195 \quad(3)$ \\
\hline Cdc85 & $0.443(3)$ & $0.5325(10)$ & $0.3500(11)$ & $0.020(3)^{*}$ & $0.195 \quad(3)$ \\
\hline Cdc86 & $0.197(4)$ & $0.7797(15)$ & $0.8615(16)$ & $0.020(3)^{*}$ & $0.195 \quad(3)$ \\
\hline
\end{tabular}


Table 4

Fractional atomic coordinates and isotropic or equivalent isotropic displacement parameters (̊2)

\begin{tabular}{|c|c|c|c|c|c|}
\hline & $x$ & $y$ & $z$ & $U_{\text {iso }} * / U_{\text {eq }}$ & Occ. $(<1)$ \\
\hline Sb1_1 & 1.600263 & $0.8787(3)$ & 0.692587 & $0.00971(9)^{*}$ & \\
\hline $\mathrm{Sb} 1 \_2$ & $2.1016(11)$ & $0.6281(3)$ & $0.6908(4)$ & $0.00971(9)^{*}$ & \\
\hline Sb1_3 & $1.6020(10)$ & $0.8707(3)$ & $0.3601(4)$ & $0.00971(9)^{*}$ & \\
\hline Sb1_4 & 1.6033 (11) & $0.6184(3)$ & $0.8548(4)$ & $0.00971(9)^{*}$ & \\
\hline Sb1_5 & $2.1013(10)$ & $0.8724(3)$ & $0.5231(4)$ & $0.00971(9)^{*}$ & \\
\hline Sb1_6 & $2.1013(11)$ & $1.1274(3)$ & $0.3551(4)$ & $0.00971(9)^{*}$ & \\
\hline Sb1_7 & 1.1033 (11) & $0.6225(3)$ & $1.0285(5)$ & $0.00971(9)^{*}$ & \\
\hline Sb1_8 & $1.5946(10)$ & $1.1261(3)$ & $0.5216(4)$ & $0.00971(9)^{*}$ & \\
\hline Sb1_9 & $1.1053(10)$ & $1.3725(3)$ & $0.5243(4)$ & $0.00971(9)^{*}$ & \\
\hline Sb1_10 & $1.6009(9)$ & $1.3639(2)$ & $0.3562(4)$ & $0.00971(9)^{*}$ & \\
\hline $\mathrm{Sb} 1 \_11$ & 1.0994 (10) & $1.1171(3)$ & $0.6883(4)$ & $0.00971(9)^{*}$ & \\
\hline Sb1_12 & $1.3815(10)$ & $0.6263(3)$ & $0.7864(4)$ & $0.00971(9)^{*}$ & \\
\hline $\mathrm{Sb} 1 \_13$ & $1.3883(10)$ & $1.1249(3)$ & $0.4501(4)$ & $0.00971(9)^{*}$ & \\
\hline Sb1_14 & $1.3832(10)$ & $0.8785(3)$ & $0.6191(4)$ & $0.00971(9)^{*}$ & \\
\hline Sb1_15 & $1.3809(11)$ & $1.3726(3)$ & $0.2806(4)$ & $0.00971(9)^{*}$ & \\
\hline $\mathrm{Sb} 1 \_16$ & $1.8855(9)$ & $0.6279(3)$ & $0.6190(4)$ & $0.00971(9)^{*}$ & \\
\hline Sb1_17 & $1.8806(7)$ & $0.8751(3)$ & $0.4514(3)$ & $0.00971(9)^{*}$ & \\
\hline $\mathrm{Sb} 1 \_18$ & $0.8855(10)$ & $1.1202(3)$ & $0.6137(4)$ & $0.00971(9)^{*}$ & \\
\hline Sb1_19 & 0.8903 (10) & $0.6142(3)$ & $0.9527(4)$ & $0.00971(9)^{*}$ & \\
\hline $\mathrm{Sb} 1 \_20$ & $1.3802(10)$ & $0.8715(2)$ & $0.2841(4)$ & $0.00971(9)^{*}$ & \\
\hline $\mathrm{Sb} 1 \_21$ & $0.8828(10)$ & $1.3689(2)$ & $0.4531(4)$ & $0.00971(9)^{*}$ & \\
\hline $\mathrm{Sb} 1 \_22$ & $1.3795(10)$ & $0.3758(3)$ & $0.6173(4)$ & $0.00971(9)^{*}$ & \\
\hline Sb1_23 & $1.8835(10)$ & $1.1273(3)$ & $0.2857(4)$ & $0.00971(9)^{*}$ & \\
\hline Sb1_24 & $1.6034(10)$ & $0.3755(3)$ & $0.6894(4)$ & $0.00971(9)^{*}$ & \\
\hline $\mathrm{Sb} 2 \_1$ & $1.8145(10)$ & $0.5380(3)$ & $0.7707(4)$ & $0.00914(9)^{*}$ & \\
\hline $\mathrm{Sb} 2 \_2$ & $1.6734(10)$ & $0.7147(3)$ & $0.7069(4)$ & $0.00914(9)^{*}$ & \\
\hline $\mathrm{Sb} 2 \_3$ & $1.4954(10)$ & $0.9490(3)$ & $0.4858(4)$ & $0.00914(9)^{*}$ & \\
\hline
\end{tabular}




\begin{tabular}{|c|c|c|c|c|}
\hline $\mathrm{Sb} 2 \_4$ & $1.3194(10)$ & $1.2828(3)$ & $0.4412(4)$ & $0.00914(9)^{*}$ \\
\hline $\mathrm{Sb} 2 \_5$ & $1.3191(10)$ & $0.7144(3)$ & $0.6113(4)$ & $0.00914(9)^{*}$ \\
\hline $\mathrm{Sb} 2 \_6$ & $1.8140(10)$ & $0.9630(3)$ & $0.6022(4)$ & $0.00914(9)^{*}$ \\
\hline $\mathrm{Sb} 2 \_7$ & $1.4896(9)$ & $0.5437(2)$ & $0.9869(4)$ & $0.00914(9) *$ \\
\hline $\mathrm{Sb} 2 \_8$ & $2.1679(10)$ & $0.5343(3)$ & $0.8671(4)$ & $0.00914(9)^{*}$ \\
\hline $\mathrm{Sb} 2 \_9$ & $1.9871(10)$ & $0.8046(3)$ & $0.6537(4)$ & $0.00914(9)^{*}$ \\
\hline $\mathrm{Sb} 2 \_10$ & $1.3206(9)$ & $0.7924(2)$ & $0.7659(4)$ & $0.00914(9)^{*}$ \\
\hline $\mathrm{Sb} 2 \_11$ & $1.3225(10)$ & $1.2098(3)$ & $0.6043(4)$ & $0.00914(9)^{*}$ \\
\hline $\mathrm{Sb} 2 \_12$ & $1.9915(10)$ & $0.6942(3)$ & $0.8209(4)$ & $0.00914(9)^{*}$ \\
\hline $\mathrm{Sb} 2 \_13$ & $1.9927(10)$ & $1.2974(3)$ & $0.3223(4)$ & $0.00914(9)^{*}$ \\
\hline $\mathrm{Sb} 2 \_14$ & $1.4922(10)$ & $0.5572(2)$ & $0.6519(4)$ & $0.00914(9)^{*}$ \\
\hline $\mathrm{Sb} 2 \_15$ & $0.9941(10)$ & $1.1981(3)$ & $0.4884(4)$ & $0.00914(9)^{*}$ \\
\hline $\mathrm{Sb} 2 \_16$ & $2.1726(10)$ & $0.5430(3)$ & $0.5407(4)$ & $0.00914(9)^{*}$ \\
\hline $\mathrm{Sb} 2 \_17$ & $1.6776(10)$ & $0.7822(2)$ & $0.5385(4)$ & $0.00914(9)^{*}$ \\
\hline $\mathrm{Sb} 2 \_18$ & $1.2949(9)$ & $0.7162(2)$ & $0.9470(4)$ & $0.00914(9)^{*}$ \\
\hline $\mathrm{Sb} 2 \_19$ & $1.4859(9)$ & $1.0492(3)$ & $0.6524(4)$ & $0.00914(9)^{*}$ \\
\hline $\mathrm{Sb} 2 \_20$ & $1.4967(10)$ & $0.4528(3)$ & $0.8180(4)$ & $0.00914(9)^{*}$ \\
\hline $\mathrm{Sb} 2 \_21$ & $1.8176(10)$ & $1.0415(2)$ & $0.4362(4)$ & $0.00914(9)^{*}$ \\
\hline $\mathrm{Sb} 2 \_22$ & $1.6831(9)$ & $0.7830(2)$ & $0.8674(4)$ & $0.00914(9)^{*}$ \\
\hline $\mathrm{Sb} 2 \_23$ & $1.4906(10)$ & $1.0496(3)$ & $0.3188(4)$ & $0.00914(9)^{*}$ \\
\hline Sb2_24 & $2.1639(10)$ & $0.9674(3)$ & $0.6971(4)$ & $0.00914(9)^{*}$ \\
\hline $\mathrm{Sb} 2 \_25$ & $1.6725(10)$ & $1.2883(3)$ & $0.5369(4)$ & $0.00914(9)^{*}$ \\
\hline $\mathrm{Sb} 2 \_26$ & $1.8005(9)$ & $0.9594(2)$ & $0.2740(4)$ & $0.00914(9)^{*}$ \\
\hline Sb2_27 & $1.8254(10)$ & $0.4678(3)$ & $0.9445(4)$ & $0.00914(9)^{*}$ \\
\hline $\mathrm{Sb} 2 \_28$ & $0.9950(10)$ & $1.3031(3)$ & $0.6531(4)$ & $0.00914(9) *$ \\
\hline $\mathrm{Sb} 2 \_29$ & $1.4973(10)$ & $1.4476(3)$ & $0.4870(4)$ & $0.00914(9)^{*}$ \\
\hline $\mathrm{Sb} 2 \_30$ & $2.1755(9)$ & $0.9636(2)$ & $0.3720(4)$ & $0.00914(9)^{*}$ \\
\hline $\mathrm{Sb} 2 \_31$ & $1.8169(10)$ & $0.4633(3)$ & $0.6039(4)$ & $0.00914(9)^{*}$ \\
\hline $\mathrm{Sb} 2 \_32$ & $2.1675(10)$ & $0.4685(3)$ & $0.7005(4)$ & $0.00914(9)^{*}$ \\
\hline $\mathrm{Sb} 2 \_33$ & $2.1574(9)$ & $1.0315(3)$ & $0.5308(4)$ & $0.00914(9) *$ \\
\hline
\end{tabular}




\begin{tabular}{|c|c|c|c|c|}
\hline $\mathrm{Sb} 2 \_34$ & $1.6825(10)$ & $1.2117(3)$ & $0.7008(4)$ & $0.00914(9)^{*}$ \\
\hline $\mathrm{Sb} 2 \_35$ & $1.6724(10)$ & $1.2105(3)$ & $0.3682(4)$ & $0.00914(9)^{*}$ \\
\hline $\mathrm{Sb} 2 \_36$ & $1.8146(10)$ & $1.0385(3)$ & $0.7746(4)$ & $0.00914(9)^{*}$ \\
\hline Cd1_1 & $1.5635(10)$ & $0.7963(3)$ & $0.7566(4)$ & $0.01184(7)^{*}$ \\
\hline Cd1_2 & $2.1002(10)$ & $0.7248(3)$ & $0.7307(4)$ & $0.01184(7)^{*}$ \\
\hline Cd1_3 & $2.0592(10)$ & $0.5506(3)$ & $0.7592(4)$ & $0.01184(7)^{*}$ \\
\hline Cd1_4 & $1.6154(11)$ & $0.9725(3)$ & $0.4001(4)$ & $0.01184(7)^{*}$ \\
\hline Cd1_5 & $1.5690(11)$ & $1.2043(3)$ & $0.5901(4)$ & $0.01184(7)^{*}$ \\
\hline Cd1_6 & $1.7726(10)$ & $0.6485(3)$ & $0.7918(4)$ & $0.01184(7)^{*}$ \\
\hline Cd1_7 & $2.0572(10)$ & $1.0458(3)$ & $0.4222(4)$ & $0.01184(7)^{*}$ \\
\hline Cd1_8 & $1.6189(11)$ & $0.5202(3)$ & $0.9020(4)$ & $0.01184(7)^{*}$ \\
\hline Cd1_9 & $1.1108(10)$ & $1.2736(3)$ & $0.5645(4)$ & $0.01184(7)^{*}$ \\
\hline Cd1_10 & $2.0588(10)$ & $0.9515(3)$ & $0.5902(4)$ & $0.01184(7)^{*}$ \\
\hline Cd1_11 & $1.6095(10)$ & $1.0262(3)$ & $0.5621(4)$ & $0.01184(7)^{*}$ \\
\hline Cd1_12 & $1.7657(11)$ & $0.8539(3)$ & $0.6272(4)$ & $0.01184(7)^{*}$ \\
\hline Cd1_13 & $1.7667(10)$ & $1.1466(3)$ & $0.4604(4)$ & $0.01184(7)^{*}$ \\
\hline Cd1_14 & $1.6052(10)$ & $0.4772(3)$ & $0.7275(4)$ & $0.01184(7)^{*}$ \\
\hline Cd1_15 & $1.4185(10)$ & $0.6997(3)$ & $0.7182(4)$ & $0.01184(7)^{*}$ \\
\hline Cd1_16 & $1.8692(11)$ & $0.7752(3)$ & $0.7410(4)$ & $0.01184(7)^{*}$ \\
\hline Cd1_17 & $1.5718(10)$ & $0.7945(3)$ & $0.4318(4)$ & $0.01184(7)^{*}$ \\
\hline Cd1_18 & $1.2527(10)$ & $1.0928(3)$ & $0.6215(4)$ & $0.01184(7)^{*}$ \\
\hline Cd1_19 & $1.3769(10)$ & $0.4753(3)$ & $0.9088(4)$ & $0.01184(7)^{*}$ \\
\hline Cd1_20 & $1.5646(10)$ & $1.2907(3)$ & $0.4292(4)$ & $0.01184(7)^{*}$ \\
\hline Cd1_21 & $1.3786(10)$ & $1.0205(3)$ & $0.4121(4)$ & $0.01184(7)^{*}$ \\
\hline Cd1_22 & $1.2561(10)$ & $0.5872(2)$ & $0.9637(4)$ & $0.01184(7)^{*}$ \\
\hline Cd1_23 & $1.0672(10)$ & $0.5431(3)$ & $1.0977(4)$ & $0.01184(7)^{*}$ \\
\hline Cd1_24 & $2.2746(10)$ & $0.6079(3)$ & $0.6310(4)$ & $0.01184(7)^{*}$ \\
\hline Cd1_25 & $1.2759(11)$ & $1.3942(3)$ & $0.4591(4)$ & $0.01184(7)^{*}$ \\
\hline Cd1_26 & $1.7579(10)$ & $1.3484(3)$ & $0.2862(4)$ & $0.01184(7)^{*}$ \\
\hline Cd1_27 & $2.2815(10)$ & $0.8932(3)$ & $0.4647(4)$ & $0.01184(7)^{*}$ \\
\hline
\end{tabular}




\begin{tabular}{|c|c|c|c|c|}
\hline Cd1_28 & 0.8713 & $1.2241(3)$ & $0.5735(4)$ & $0.01184(7)^{*}$ \\
\hline Cd1_29 & $2.1168(11)$ & $1.2258(3)$ & $0.4024(4)$ & $0.01184(7)^{*}$ \\
\hline Cd1_30 & $1.7141(10)$ & $0.6054(3)$ & $0.6828(4)$ & $0.01184(7)^{*}$ \\
\hline Cd1_31 & $1.9173(11)$ & $0.5435(3)$ & $0.8771(4)$ & $0.01184(7)^{*}$ \\
\hline Cd1_32 & $2.1222(10)$ & $0.7778(3)$ & $0.5692(4)$ & $0.01184(7)^{*}$ \\
\hline Cd1_33 & $1.3695(10)$ & $0.9761(3)$ & $0.5711(4)$ & $0.01184(7)^{*}$ \\
\hline Cd1_34 & $1.9114(10)$ & $0.9537(3)$ & $0.3821(4)$ & $0.01184(7)^{*}$ \\
\hline Cd1_35 & $1.2122(11)$ & $1.1433(3)$ & $0.5122(4)$ & $0.01184(7)^{*}$ \\
\hline Cd1_36 & $2.2116(10)$ & $0.6425(3)$ & $0.8510(4)$ & $0.01184(7)^{*}$ \\
\hline Cd1_37 & $1.7154(10)$ & $0.8935(3)$ & $0.5165(4)$ & $0.01184(7)^{*}$ \\
\hline Cd1_38 & $1.3715(11)$ & $0.5282(3)$ & $0.7385(4)$ & $0.01184(7)^{*}$ \\
\hline Cd1_39 & $1.9138(10)$ & $0.4607(3)$ & $0.7106(4)$ & $0.01184(7)^{*}$ \\
\hline Cd1_40 & $1.4199(11)$ & $1.2975(3)$ & $0.5487(4)$ & $0.01184(7)^{*}$ \\
\hline Cd1_41 & $1.6122(11)$ & $0.9738(3)$ & $0.7342(4)$ & $0.01184(7)^{*}$ \\
\hline Cd1_42 & $1.8736(10)$ & $1.2664(3)$ & $0.4129(4)$ & $0.01184(7)^{*}$ \\
\hline Cd1_43 & $2.2105(11)$ & $0.8567(3)$ & $0.6806(4)$ & $0.01184(7)^{*}$ \\
\hline Cd1_44 & $1.9139(10)$ & 1.0409 (3) & $0.5429(4)$ & $0.01184(7)^{*}$ \\
\hline Cd1_45 & $1.0651(11)$ & $1.4503(3)$ & $0.5920(4)$ & $0.01184(7)^{*}$ \\
\hline Cd1_46 & $1.2304(10)$ & $0.6545(3)$ & $0.5238(4)$ & $0.01184(7)^{*}$ \\
\hline Cd1_47 & $1.1227(10)$ & $0.7244(3)$ & $1.0681(4)$ & $0.01184(7)^{*}$ \\
\hline Cd1_48 & $1.2311(10)$ & $1.3450(3)$ & $0.3490(4)$ & $0.01184(7)^{*}$ \\
\hline Cd1_49 & $2.2636(10)$ & $1.1038(3)$ & $0.2902(4)$ & $0.01184(7)^{*}$ \\
\hline Cd1_50 & $1.0628(10)$ & $1.0397(3)$ & $0.7629(4)$ & $0.01184(7)^{*}$ \\
\hline Cd1_51 & $1.3839(10)$ & $0.5214(3)$ & $1.0806(4)$ & $0.01184(7)^{*}$ \\
\hline Cd1_52 & $1.7146(10)$ & $0.8900(3)$ & $0.1839(4)$ & $0.01184(7)^{*}$ \\
\hline Cd1_53 & $1.4226(11)$ & $1.2031(3)$ & $0.3814(4)$ & $0.01184(7)^{*}$ \\
\hline Cd1_54 & $1.7571(10)$ & $0.8392(3)$ & $0.2948(4)$ & $0.01184(7)^{*}$ \\
\hline Cd1_55 & $1.7707(10)$ & $0.3493(3)$ & $0.6286(4)$ & $0.01184(7)^{*}$ \\
\hline Cd1_56 & $1.7385(10)$ & $0.4079(3)$ & $0.8564(4)$ & $0.01184(7)^{*}$ \\
\hline Cd1_57 & $1.7153(11)$ & $1.1034(3)$ & $0.3492(4)$ & $0.01184(7)^{*}$ \\
\hline
\end{tabular}




\begin{tabular}{|c|c|c|c|c|c|}
\hline Cd1_58 & $1.8678(11)$ & $1.2270(3)$ & $0.2407(4)$ & $0.01184(7)^{*}$ & \\
\hline Cd1_59 & $1.9093(10)$ & $0.9604(3)$ & $0.7092(4)$ & $0.01184(7)^{*}$ & \\
\hline Cd1_60 & $1.3830(10)$ & $0.4791(3)$ & $0.5771(4)$ & $0.01184(7)^{*}$ & \\
\hline Cd1_61 & $1.7145(10)$ & $1.3953(3)$ & $0.5174(4)$ & $0.01184(7)^{*}$ & \\
\hline Cd1_62 & $1.9246(10)$ & $0.5459(3)$ & $0.5540(4)$ & $0.01184(7)^{*}$ & \\
\hline Cd1_63 & $1.7324(10)$ & $1.0960(3)$ & $0.6808(4)$ & $0.01184(7)^{*}$ & \\
\hline Cd1_64 & $1.4315(10)$ & $0.8005(3)$ & $0.5531(4)$ & $0.01184(7)^{*}$ & \\
\hline $\mathrm{Cd} 3 \_1$ & $0.9614(9)$ & $1.2057(3)$ & $0.7031(4)$ & $0.0041(3)^{*}$ & $0.836 \quad$ (3) \\
\hline $\mathrm{Cd} 3 \_2$ & $0.5007(9)$ & $1.3497(3)$ & $0.7765(4)$ & $0.0041(3)^{*}$ & $0.836 \quad$ (3) \\
\hline $\mathrm{Cd} 3 \_3$ & $1.0450(10)$ & $1.2938(3)$ & $0.7696(4)$ & $0.0041(3)^{*}$ & $0.836 \quad$ (3) \\
\hline $\mathrm{Cd} 3 \_4$ & $1.2582(9)$ & 1.1997 (2) & $0.7142(4)$ & $0.0041(3)^{*}$ & $0.836 \quad$ (3) \\
\hline $\mathrm{Cd} 3 \_5$ & $1.5004(9)$ & $1.1465(2)$ & $0.7043(4)$ & $0.0041(3)^{*}$ & $0.836 \quad(3)$ \\
\hline $\mathrm{Cd} 3 \_6$ & $0.4658(9)$ & $1.2754(2)$ & $0.6948(4)$ & $0.0041(3)^{*}$ & 0.836 \\
\hline $\mathrm{Cd} 3 \_7$ & $0.7761(10)$ & $1.2757(3)$ & $0.7895(4)$ & $0.0041(3)^{*}$ & 0.836 \\
\hline $\mathrm{Cd} 3 \_8$ & $1.0414(10)$ & $0.7067(3)$ & $0.9408(4)$ & $0.0041(3)^{*}$ & 0.836 \\
\hline Cd3_9 & $1.4547(10)$ & $1.4556(3)$ & $0.3702(4)$ & $0.0041(3)^{*}$ & $0.836 \quad$ (3) \\
\hline Cd3_10 & $1.5196(9)$ & $0.6517(2)$ & $0.9520(4)$ & $0.0041(3)^{*}$ & $0.836 \quad(3)$ \\
\hline Cd3_11 & $1.4528(9)$ & $0.8474(3)$ & $0.8538(4)$ & $0.0041(3)^{*}$ & $0.836 \quad(3)$ \\
\hline $\mathrm{Cd} 3 \_12$ & $1.4659(9)$ & $0.7251(2)$ & $0.8634(4)$ & $0.0041(3)^{*}$ & $0.836 \quad(3)$ \\
\hline $\mathrm{Cd} 3 \_13$ & $1.2497(9)$ & $0.7987(2)$ & $0.8785(4)$ & $0.0041(3)^{*}$ & $0.836 \quad(3)$ \\
\hline Cd3_14 & $1.2711(9)$ & $0.5312(2)$ & $0.4512(4)$ & $0.0041(3)^{*}$ & $0.836 \quad$ (3) \\
\hline $\mathrm{Cd} 2 \_1$ & $0.527(4)$ & $1.3220(15)$ & $0.7817(15)$ & $0.021(3)^{*}$ & $0.164 \quad(3)$ \\
\hline $\mathrm{Cd} 2 \_2$ & $1.379(3)$ & 1.2755 (12) & $0.7222(13)$ & $0.021(3)^{*}$ & $0.164 \quad(3)$ \\
\hline $\mathrm{Cd} 2 \_3$ & $0.951(3)$ & $1.2668(12)$ & $0.7228(13)$ & $0.021(3)^{*}$ & $0.164 \quad(3)$ \\
\hline $\mathrm{Cd} 2 \_4$ & $1.031(3)$ & $1.2274(12)$ & $0.7788(13)$ & $0.021(3)^{*}$ & $0.164 \quad(3)$ \\
\hline $\mathrm{Cd} 2 \_5$ & $-1.498(3)$ & $0.6780(14)$ & $-0.0718(14)$ & $0.021(3)^{*}$ & $0.164 \quad(3)$ \\
\hline $\mathrm{Cd} 2 \_6$ & $0.387(3)$ & $0.8133(12)$ & $0.8466(14)$ & $0.021(3)^{*}$ & $0.164 \quad(3)$ \\
\hline $\mathrm{Cd} 2 \_7$ & $0.225(3)$ & $0.5027(12)$ & $0.3892(13)$ & $0.021(3)^{*}$ & $0.164 \quad(3)$ \\
\hline $\mathrm{Cd} 2 \_8$ & $0.199(4)$ & $0.7733(17)$ & $0.8593(17)$ & $0.021(3)^{*}$ & $0.164 \quad(3)$ \\
\hline
\end{tabular}


${ }^{i}$ Caillat, T.; Fleurial, J.-P.; Borshchevsky, A. J. Phys. Chem. Solids 1997, 58, 1119.

${ }^{\text {ii }}$ Snyder, G. J.; Christensen, M.; Nishibori, E.; Caillat, T.; Iversen, B. B. Nat. Mater. 2004, 3, 458.

iii Nylén, J.; Andersson, M.; Lidin, S.; Häussermann, U. J. Am. Chem. Soc. 2004, 126,16306.

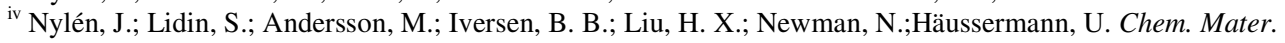
2007, 19, 834.

${ }^{v}$ Nylén; J.; Lidin, S.; Andersson, M.; Liu, H.; Newman, N.; Häussermann, U. J. Solid State Chem. 2007, 180, 2603.

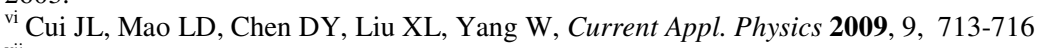

vii Pedersen, B.L. Birkedal, H., Nygren, M. Fredriksen, P.T and Iversen B.B, J Appl. Phys. 2009, 105, 013517

viii Nakamoto G, Kinoshita K, Kurisu M, J Appl Phys, 2009, 105, 013713

${ }^{i x}$ Litvinchuk AP, Nylen J, Lorenz B, Guloy, AM and Häussermann, U. J. Appl Phys. 2008, 103, 123524

${ }^{x}$ Tengå, A.; Lidin, S.; Belieres, J.-P.; Newman, N.; Yang, W. and Häußermann, U. JACS, asap 2009

xi Petricek, V., Dusek, M. And Palatinus, L.Institute of Physics, Academy of Sciences of the Czech Republic, Praha, 2005

xii Zelinska, O. Y.; Bie, H. Y.; Mar, A. Chem. Mater. 2007, 19, 1518. 


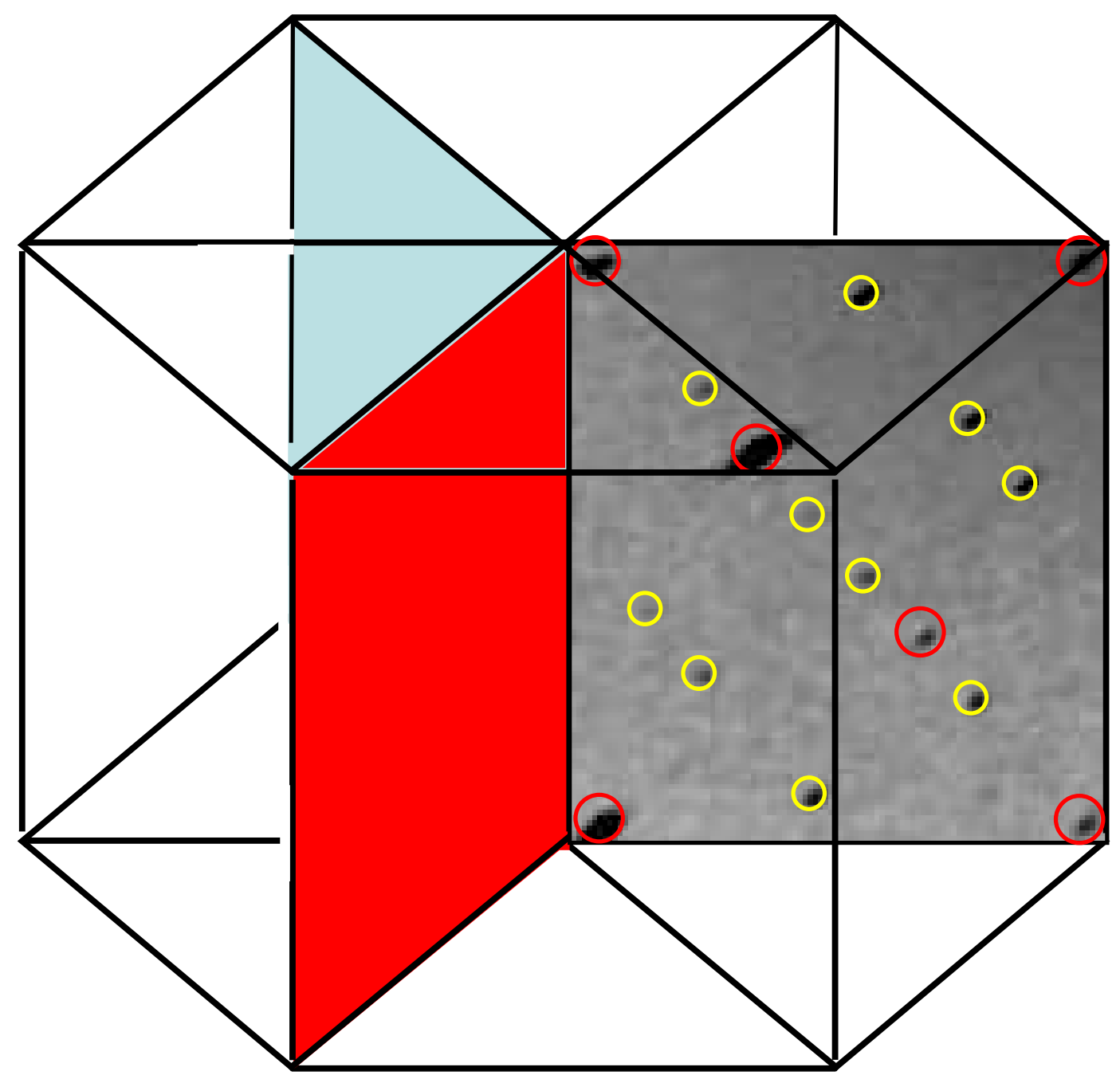

Fig 1 


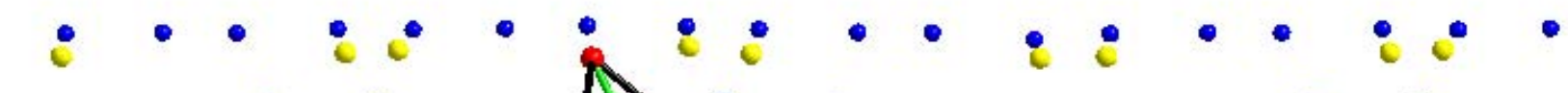

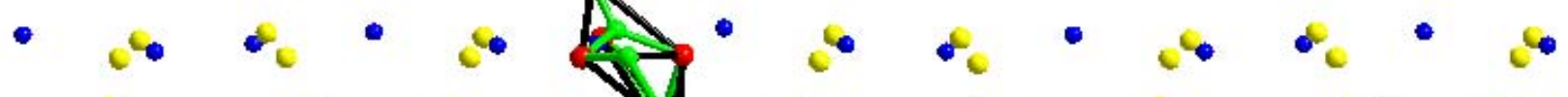
$e^{\circ} \cdot e^{\circ} \cdot e^{\circ} \cdot e^{\circ} \cdot e^{\circ}$

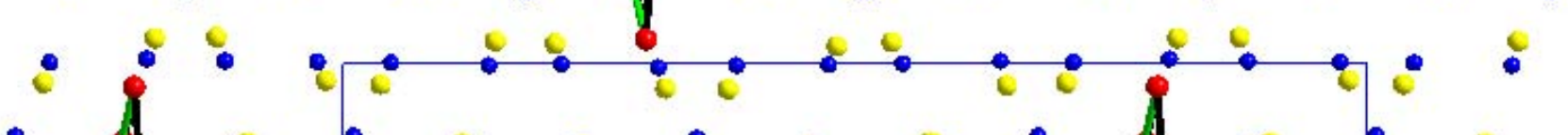

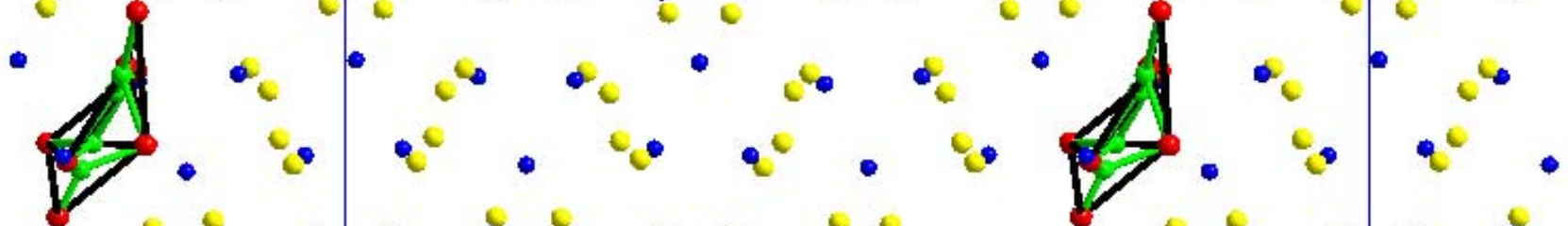

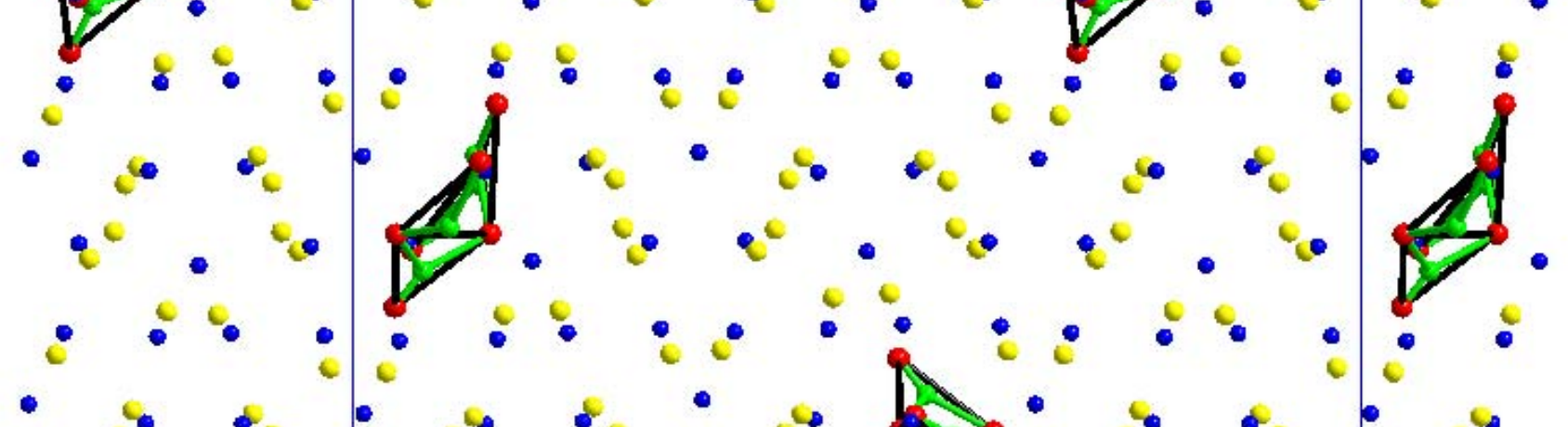

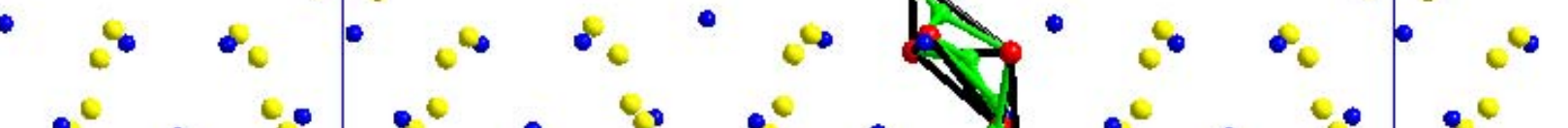
$e^{\circ} \cdot e^{\circ} \cdot 4 e^{\circ} \cdot e^{\circ} \cdot e^{\circ}$ $\div: \div \div \div \div \div \div \div \div \div \div$

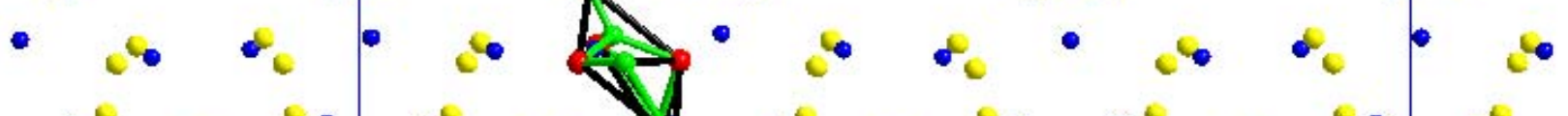
$e^{\circ} \cdot e^{\circ} \cdot 0^{\circ} \cdot e^{\circ} \cdot e^{\circ} \cdot e^{\circ}$

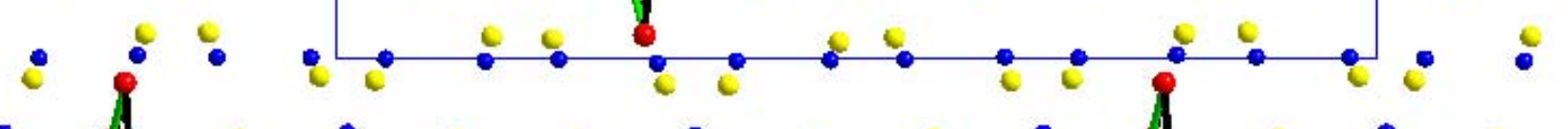

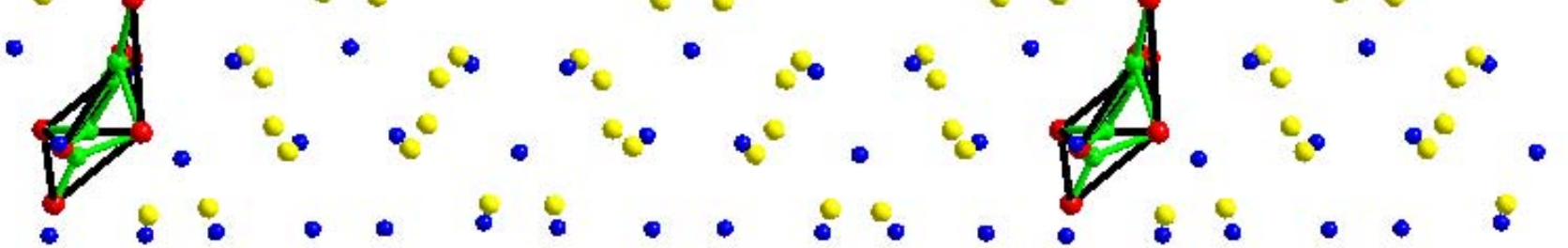
Fig 2a 


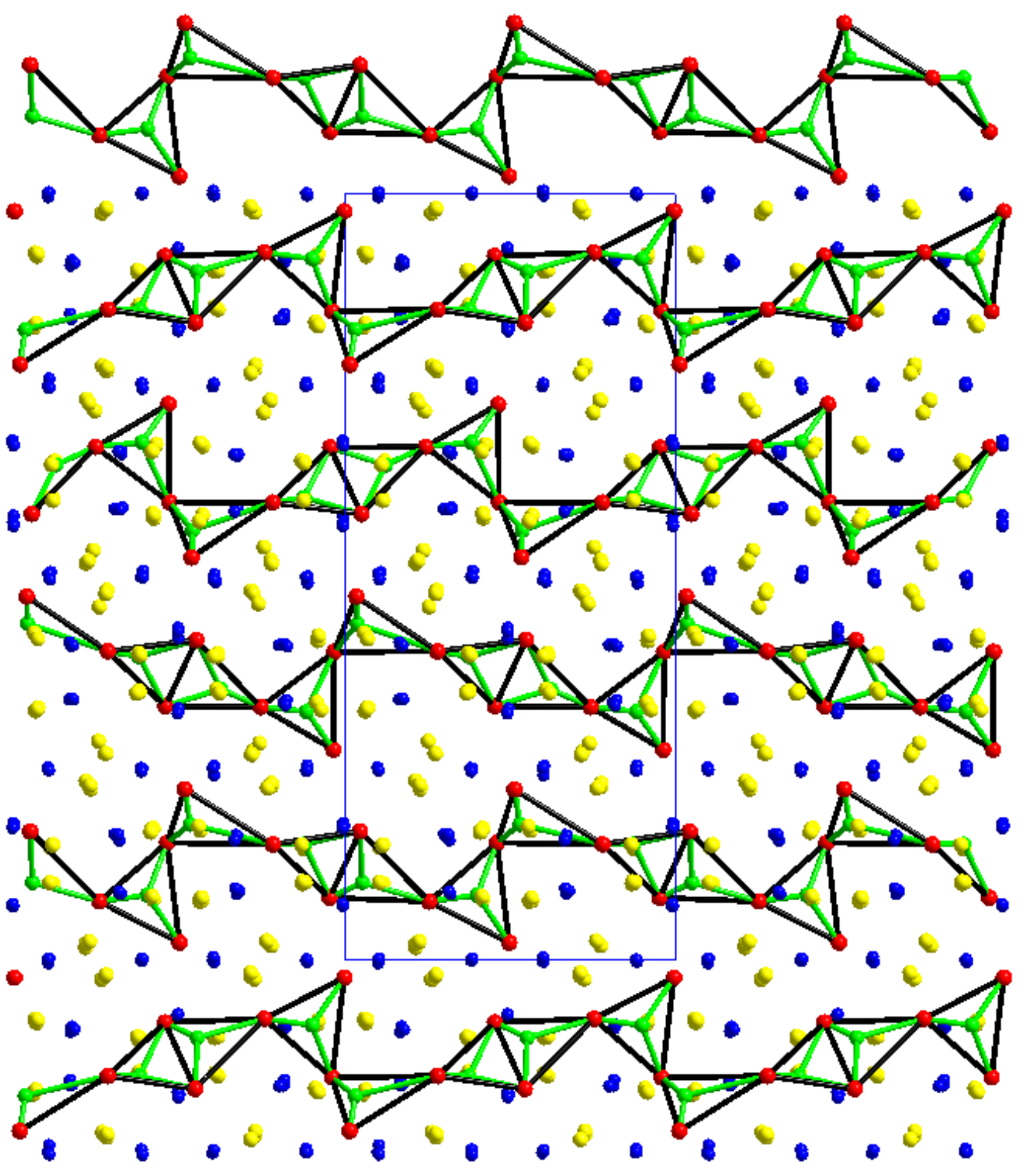

Fig2b 


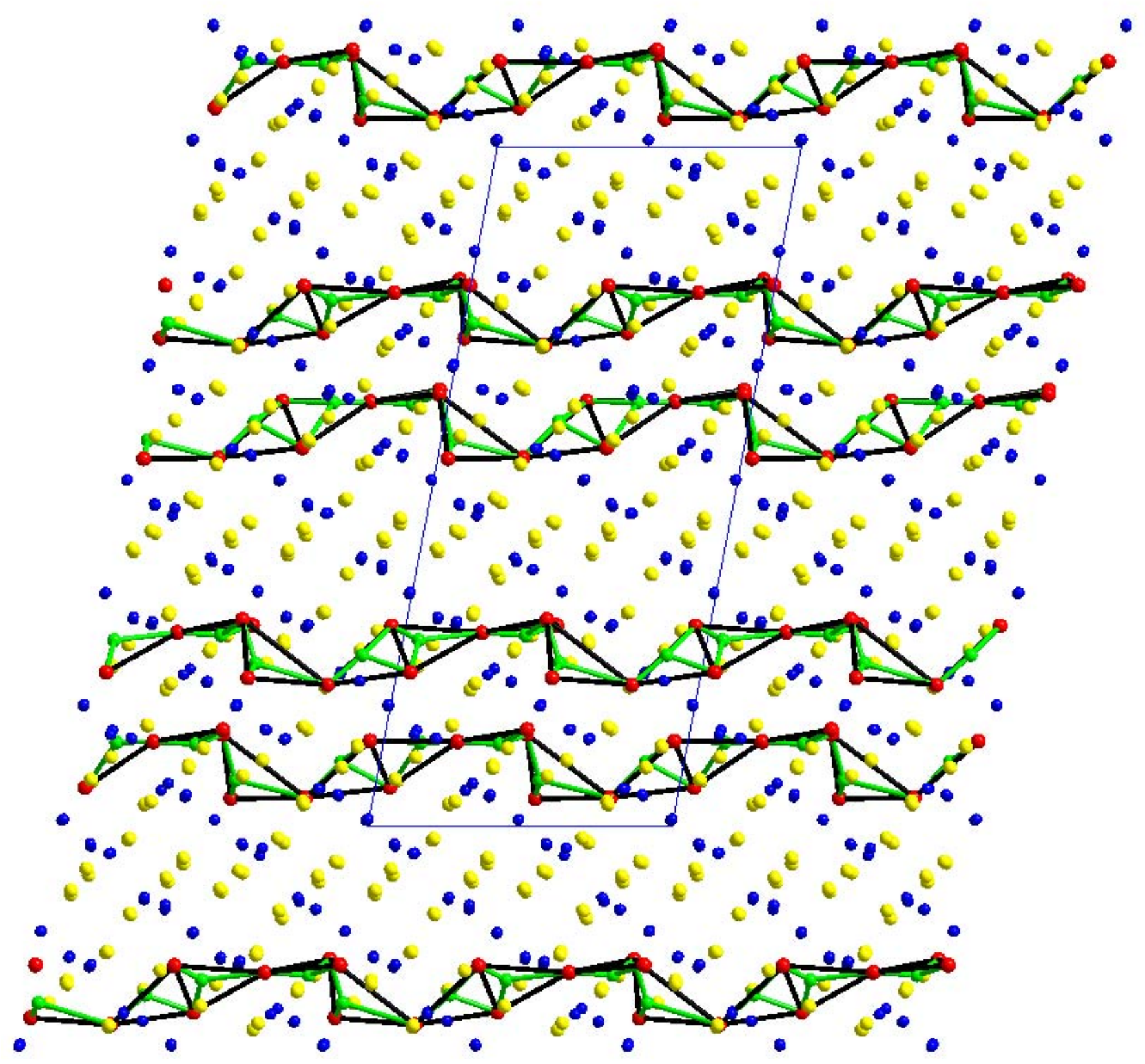

Fig 2c 
5

6
7

8

9

10

11

12

13

14

15

16

17

18

19

20

22

23

24

25

26

27

29

30

31

32

33

34

35

36

37

38

39

40

41

42

43

44

45

46

47

48

49

50

51

52

53

54

55

56

57

58

59 